
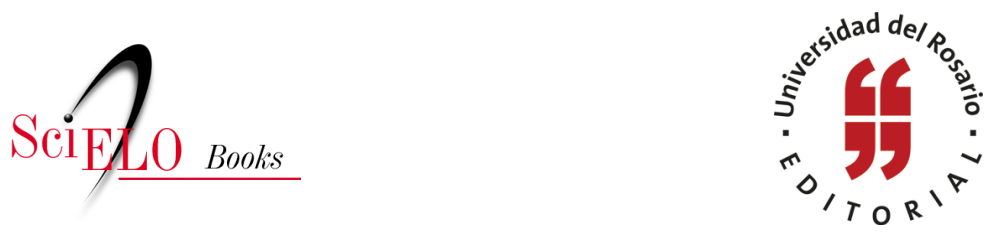

\title{
Construcción de la política cultural en la Universidad del Rosario
}

\author{
Adriana Díaz Támara \\ Margarita Guzmán Bejarano \\ Ramón E. Villamizar Maldonado
}

\section{SciELO Books / SciELO Livros / SciELO Libros}

DÍAZ TÁMARA, A., GUZMÁN BEJARANO, M., and VILLAMIZAR MALDONADO, R.E. Construcción de la política cultural en la Universidad del Rosario. In: DÍAZ TÁMARA, A., and GUZMÁN BEJARANO, M., eds. Proceso de construcción de la política cultural en la Universidad del Rosario [online]. Bogotá: Editorial Universidad del Rosario, Decanatura del Medio Universitario, 2014, pp. 11-26. ISBN: 978-958-738-541-0. https://doi.org/10.7476/9789587385410.0001.

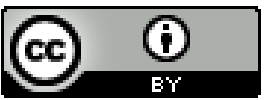

All the contents of this work, except where otherwise noted, is licensed under a Creative Commons Attribution 4.0 International license.

Todo o conteúdo deste trabalho, exceto quando houver ressalva, é publicado sob a licença Creative Commons Atribição 4.0.

Todo el contenido de esta obra, excepto donde se indique lo contrario, 


\title{
Construcción de la política cultural en la Universidad del Rosario
}

\author{
Adriana Díaz Támara* \\ Margarita Guzmán Bejarano** \\ Ramón E. Villamizar Maldonado***
}

Este documento pretende iniciar un proceso de reflexión, construcción colectiva y concertación de una política cultural en la Universidad del Rosario, que permita definir qué entiende la institución por cultura, enmarcarla dentro de su misión institucional y establecer unas líneas de acción, programas y objetivos que hagan posible su sostenibilidad, así como mecanismos y acciones de acompañamiento que den paso a la implementación y evaluación del quehacer cultural en el Rosario.

El compromiso con los criterios de pluralidad e inclusión impone definir acciones estratégicas que permitan la adopción de normas que favorezcan la actividad cultural y así crear y participar de una red cultural que se inserte a nivel local y nacional con el fin de promover la implementación y la consolidación de prácticas culturales.

* Abogada por la Universidad del Rosario, coordinadora académica y cultural de la Decanatura del Medio Universitario, Universidad del Rosario.

**A Artista, museóloga y gestora de patrimonio, profesora principal de carrera académica, Decanatura del Medio Universitario, Universidad del Rosario.

**** Politólogo por la Universidad de los Andes, especialista en Gobierno y Políticas Públicas, docente de la Especialización en Gerencia y Gestión Cultural de la Universidad del Rosario. 
Ante la pregunta ¿para qué una política cultural en la Universidad del Rosario?, se reconoce que se diseña una política en la Universidad del Rosario con cinco propósitos: fortalecer la apropiación del patrimonio y la cultura rosarista; desarrollar los valores democráticos de la comunidad universitaria; aportar a la formación integral de los estudiantes; estimular la creatividad; asumir el conocimiento como diversidad y pluralidad de saberes; y afianzar los proyectos de responsabilidad social con los que se encuentra comprometida la institución.

En este ejercicio de construcción fue fundamental la identificación de fuentes importantes para el diseño de la política. En primer lugar, se revisaron documentos marco, como la Declaración de la Unesco sobre Educación Superior, la Ley General de Cultura (Ley 397 de $1997^{1}$ y Ley 1185 de 2008²); la Ley 30 de 1992; ${ }^{3}$ el Sistema Distrital de Arte, Cultura y Patrimonio (Decretos 627 de 2007, 083 de $2008^{4}$ y 455 de $2009^{5}$ ); las políticas culturales distritales $2004-2016^{6}$ y la discusión sobre las políticas y

1 Ver: http://www.sinic.gov.co/SINIC/Sipa_Conceptos_Comite_Tecnico/ley\%20397\%20de\%201997.pdf febrero 21 de 2013, 10:15 am.

2 Ver: http://www.secretariasenado.gov.co/senado/basedoc/ ley/2008/ley_1185_2008.html febrero 21 de 2013, 10:15 am.

3 Ver: http://www.secretariasenado.gov.co/senado/basedoc/ ley/1992/ley_0030_1992.html febrero 21 de 2013, 10:15 am.

4 Ver: http://www.culturarecreacionydeporte.gov.co/cultura_ recreacion_y_deporte/sistema_cultura_arte_y_patrimonio/descargas/Decreto \%20627\%20de\%202007.pdf febrero 21 de 2013, 10:15 am.

5 Ver: http://www.culturarecreacionydeporte.gov.co/portal/sites/ default/files/DOCUMENTO \%20FINAL \%20REGLAMENTO \% 20CLACP\%20 USAQUEN.pdf febrero 21 de 2013, 10:15 am.

6 Ver: http://www.culturarecreacionydeporte.gov.co/portal/sites/ default/files/1/politicas/1.pdf febrero 21 de 2013, 10:15 am. 
los derechos culturales ${ }^{7}$ promovida en Bogotá por la Secretaría de Cultura, Recreación y Deporte. Igualmente, se consultó el Proyecto Educativo Institucional (PEI) de la Universidad del Rosario, el Plan Integral de Desarrollo 2004-2019, y se revisó el concepto de formación integral de la institución. También fueron analizados varios documentos sobre política cultural universitaria en Colombia, principalmente el plan de Cultura de la Universidad de Antioquia "La cultura: fundamento de una universidad pertinente" (2006-2016) y el documento de trabajo de la Mesa Cultura de Instituciones de Educación Superior de Antioquia: "Hacia la construcción de una política nacional de cultura para la educación superior: un nuevo pacto social desde la cultura"8 (Medellín, junio de 2008). De la misma manera se consultaron las conclusiones del IV Encuentro Nacional de Políticas Culturales Universitarias que se llevó a cabo en abril del 2012 en Medellín. ${ }^{9}$ Dicho encuentro tuvo como objetivo promover una reflexión respecto a los fundamentos que deben tenerse en cuenta para el diseño de una política cultural para las instituciones de educación superior. Allí se acordó que era necesario concertar unos criterios generales para la formulación de políticas culturales universitarias que tengan como objetivo revisar el papel de estas instituciones como proyecto cultural de la sociedad, con el fin de lograr un diálogo con entes guberna-

7 ALCALDÍA DE BOGOTÁ, SECRETARÍA DE CULTURA, RECREACIÓNY DEPORTE DE BOGOTÁ, CORPORACIÓN CENTRO DE ESTUDIOS DE DERECHO, JUSTICIAY SOCIEDAD (DE JUSTICIA). Derechos culturales en la ciudad. Bogotá: Alcaldía de Bogotá, 2011, p. 236.

8 Ver: http://mesaculturalantioquia.files.wordpress.com/2009/04/ ponencia_congreso_de_universidades-21_de_junio1.pdf junio 19 de 2013, 11:00 am.

Ver: http://mesaculturalantioquia.files.wordpress.com/2009/03/ relatoria-general.pdf febrero 21 de 2013, 10:15 am. 
mentales tales como los ministerios de Educación Nacional, de Cultura, de Tecnologías de la Información y Comunicaciones y con el Departamento Administrativo de Ciencia, Tecnología e Innovación (Colciencias).

Para diseñar una política cultural universitaria, las instituciones de educación superior de hoy y de mañana deberán establecer múltiples y diversos vínculos con la sociedad, cumplir funciones que impacten en el ámbito regional y local, con el objetivo de apoyar la formación de una cultura cívica y para contribuir al mejoramiento de las condiciones de vida de sus ciudadanos, en cumplimiento de su responsabilidad social, tal y como lo señala la Unesco. ${ }^{10}$ Por lo tanto, las instituciones de educación superior requieren de un replanteamiento de sus programas académicos con el fin de incluir sus propios criterios que trasciendan la formación disciplinar y que contengan aspectos relacionados con el contexto cultural, histórico y económico de cada país.

En el ámbito colombiano, la Constitución Política de 1991, ${ }^{11}$ en su artículo 70, otorga al Estado un papel muy importante en la cultura, relacionado con su promoción y fomento. Señala, además, que todos los colombianos podrán acceder a ella en igualdad de oportunidades a través de la educación para lograr la búsqueda de la identidad nacional. Así, la cultura es transformadora y es contexto de producción e interpretación de significados, le da sentido a los contenidos de los conceptos

10 UNESCO. Conferencia mundial sobre la educación superior. La educación superior en el siglo xxi, Visión y acción, 9 de octubre de 1998. Disponible en: http://www.unesco.org/education/educprog/wche/declaration_spa.htm febrero 21, 10:40 am.

11 Ver: http://www.alcaldiabogota.gov.co/sisjur/normas/Norma1. jsp?i=4125 
desplegados en la dimensión social de la identidad y la memoria de una nación en desarrollo.

La Ley General de Cultura (Ley 397 de 1997) y la Ley 1185 de marzo de 2008 reglamentan en varios artículos diversas orientaciones sobre la participación de las instituciones educativas en los procesos culturales de la Nación. Así mismo, establecen la responsabilidad del nuevo ciudadano que requiere el país y señalan los lineamientos que deben asumir las instituciones de educación superior para tal fin. Los procesos abordados sobre arte y cultura han contribuido al tema de identidad y diálogo cultural, y son un aporte de fortalecimiento y desarrollo.

A nivel del Distrito Capital, las políticas culturales distritales 2004-2016 ${ }^{12}$ fueron el resultado de un proceso de concertación en las instancias del Sistema Distrital de Cultura. Al poner el foco en las prácticas de los actores (creación, formación, investigación, circulación, apropiación) y en los procesos (organización, planificación, fomento e información), el Distrito estableció un marco (campo) de relacionamiento que articula instituciones, profesiones, disciplinas académicas, públicos en torno a dichas prácticas y procesos.

La organización de la cultura en el Distrito está reglamentada a través de los Decretos Distritales $627^{13}$ de diciembre 28 de 2007 y por el Decreto 083 de 2008, mediante los cuales se reforma el Sistema Distrital de Cultura y se establece el Sistema Distrital de Arte, Cultura y Patrimonio. El Decreto $627,{ }^{14}$ en su

12 Ver: http://www.culturarecreacionydeporte.gov.co/portal/sites/ default/files/1/politicas/1.pdf febrero 25 de 2013.

13 Ver: http://www.alcaldiabogota.gov.co/sisjur/normas/Normal. jsp?i=28164 febrero 25 de 2013.

14 Ver: http://www.alcaldiabogota.gov.co/sisjur/normas/Normal. jsp?i=37647 febrero 25 de 2013. 
artículo primero, define el Sistema Distrital de Arte, Cultura y Patrimonio como una interacción social organizada entre los agentes culturales, organismos y organizaciones que desarrollan actividades relacionadas con el arte, la cultura y el patrimonio. Las universidades entendidas como agentes culturales y espacios destinados al encuentro, deliberación, participación y concertación del desarrollo cultural de la ciudad, son fundamentales para las finalidades establecidas en dicho sistema.

Por su parte, el Plan Decenal de Cultura 2012-2021 ${ }^{15}$ reconoce que las universidades son el medio para generar y articular procesos culturales desde la investigación, igualmente les da gran importancia a la hora de consolidar la dimensión de formación, así como a la profesionalización de los actores y agentes en la ciudad.

Otro aspecto para destacar tiene que ver con los esfuerzos del Distrito por comprender e incluir la garantía y exigibilidad de los derechos culturales en las políticas culturales. Este debate tiene en la Declaración de Friburgo de 2007 uno de los referentes obligados pero, como lo señalan Uprimny y Sánchez, ${ }^{16}$ "en contraste con el creciente protagonismo político de los derechos culturales, su desarrollo jurídico ha sido lento y parcial, y en todo caso inferior al de los otros derechos humanos". ${ }^{17}$

El plan de desarrollo Bogotá Humana 2012-2016 se sustenta en el concepto de desarrollo humano entendido como "un proceso que amplía el abanico de opciones de las personas,

15 Ver: http://www.culturarecreacionydeporte.gov.co/plandecenaldecultura/Plan_\%20Decenal_cultura.pdf febrero 25 de 2013.

16 ALCALDÍA DE BOGOTÁ, SECRETARÍA DE CULTURA, RECREACIÓNY DEPORTE DE BOGOTÁ, CORPORACIÓN CENTRO DE ESTUDIOS DE DERECHO, JUSTICIAY SOCIEDAD (DE JUSTICIA). Derechos culturales en la ciudad. Bogotá: Alcaldía de Bogotá, 2011.

17 Ibid., p. 28. 
permitiendo que ellas escojan el proyecto de vida que juzgan valioso". ${ }^{18}$ Las políticas públicas deben buscar crear condiciones y oportunidades para que las personas puedan "realizar el tipo de vida que consideren valioso y deseable, basándose para ello en el conjunto de bienes que se encuentren disponibles en la sociedad, y que pueden utilizar efectivamente para el cumplimiento de sus fines. El aumento en la provisión de bienes es el fundamento de la ampliación de las capacidades. La adquisición de titularidades y los bienes de diverso tipo, permiten que las personas puedan ejercer sus derechos". ${ }^{19}$

18 Alcaldía Mayor de Bogotá. Bases del Plan de Desarrollo Bogotá Humana 2012-2016, p. 15.

19 Ibid.

Así, desde los primeros documentos y en proceso de discusión y concertación, se buscó que la cultura se integrara al plan en una doble perspectiva: "como la dimensión integradora de lo humano, base constitutiva de todo sujeto y comunidad, y como un sector específico donde se ejercen los derechos humanos culturales y deportivos y se producen manifestaciones, bienes y servicios que expresan y conforman las identidades culturales y la fisionomía de una sociedad y una época, que están siempre en permanente transformación”.

El plan fue aprobado mediante el Acuerdo 489 del 12 de junio de 2012 y está compuesto por la parte programática y tres ejes estratégicos: una ciudad que supera la segregación y la discriminación: el ser humano en el centro de las preocupaciones del desarrollo; un territorio que enfrenta el cambio climático y se ordena alrededor del agua y el tercero, una Bogotá que defiende y fortalece lo público.

En el primer eje y como estrategia (artículo 10) se reconoce "la dimensión específica de la cultura, el arte, la actividad física, la recreación y el deporte" y se formula el programa "ejercicio de las libertades culturales, recreativas y deportivas" (artículo 14) que busca "superar las barreras que limitan las oportunidades para ejercer los derechos culturales y deportivos". Como proyectos prioritarios se incluyeron los siguientes: corredores culturales y recreativos; arte, cultura y patrimonio para la transformación; cotidianidad libre y activa; ciudadanías juveniles; Bogotá: capital creativa de la música; canal capital; televisión pública para los derechos y fortalecimiento de las redes de bibliotecas y fomento o valoración a la lectura. 
La mirada transversal tiene una clara expresión en el eje II, un territorio que enfrenta el cambio climático y se ordena alrededor del agua, en los objetivos (artículo 24, numeral 4) se plantea "promover cambios culturales y facilitar las condiciones para la transformación de la ciudad. Contribuir, desde lo cultural, a la construcción y consolidación de un nuevo modelo de ciudad y formas de hacer la gestión pública, que propendan por el reconocimiento y promoción de imaginarios, representaciones, prácticas culturales y percepciones que generen comportamientos sostenibles y sustentables de ordenación y planeación del territorio que permitan superar los factores de segregación social".

Igualmente la estrategia 7 (artículo 27) busca "promover cambios culturales y facilitar las condiciones para la transformación de la ciudad. Contribuir, desde lo cultural, a la construcción y consolidación de un nuevo modelo de ciudad y formas de hacer la gestión pública, que propendan por el reconocimiento y promoción de imaginarios, representaciones, prácticas culturales y percepciones que generen comportamientos sostenibles y sustentables de ordenación y planeación del territorio que permitan superar los factores de segregación social”.

Finalmente, es importante destacar que a partir de un trabajo interinstitucional realizado con la asesoría del profesor de la Universidad Nacional Javier Sáenz, se construyó en 2012 un documento con las bases de una estrategia de cultura ciudadana a partir de una relectura de esta experiencia desde los aspectos programáticos del Plan Bogotá Humana.

El documento reconoce primero que todas las potencialidades pedagógicas y comunicativas del Plan de Desarrollo y que el cambio del modelo de ciudad por el que propende requiere "promover transformaciones culturales a través del ejercicio de nuevas formas de ciudadanía que posibiliten el bienestar común en medio de la diversidad. Las nuevas ciudadanías pueden considerarse como nuevas formas de ver, sentir, pensar y actuar en lo público. Formas basadas en el reconocimiento de la diversidad cultural, la opción de disentir, la multiplicidad de proyectos de vida y la construcción de acuerdos mínimos para mejorar el orden social. Esto implica un anclaje en las prácticas culturales de las personas, entendidas como aquellas acciones que movilizan saberes, valores, imaginarios, hábitos y actitudes que suceden en lo público”.

El propósito central de la estrategia sería entonces "la ampliación, en el largo plazo, de un conjunto de capacidades políticas y culturales (definidas en términos de conocimientos, comportamientos y representaciones) de la población y de los funcionarios del gobierno de la ciudad, que potencien un ejercicio más democrático, creativo, valeroso, colectivo, decidido y constante de sus libertades y derechos sociales, económicos, políticos y culturales. Se trata 


\section{La propuesta cultural del Plan de Desarrollo Bogotá}

Humana 2012-2016 se sustentó en los postulados de Amartya Sen. Este autor plantea que "la cultura debe ser considerada en grande, no como un simple medio para alcanzar ciertos fines, sino como la misma base social [del desarrollo]. ${ }^{20}$ Igualmente, se recogió el informe PNUD de Desarrollo Humano para Bogotá (2008) que expuso que "la cultura es un campo estratégico por su proyección, es intersectorial desde la mirada de las políticas públicas y es propicia para el desarrollo humano porque favorece la ampliación de las libertades". ${ }^{21}$

de apostarle a la ampliación del "abanico de opciones de las personas, permitiendo que ellas escojan el proyecto de vida que juzgan valioso". Finalmente el documento propone denominar la estrategia como "cultura democrática" y no cultura ciudadana. En el momento de escribir este artículo este aspecto sigue sin lograr un consenso al interior de la Administración.

La definición de las acciones concretas es responsabilidad de cada sector de la Administración y entre las experiencias en curso en 2013 se destaca la estrategia de "cultura ambiental" liderada por la Secretaría Distrital de Ambiente y cuyo objetivo es "generar oportunidades y aumentar las capacidades éticas, políticas y culturales de los individuos y grupos sociales para que en el ejercicio de sus libertades, transformen los conocimientos, comportamientos y representaciones en torno a lo ambiental y fortalezcan las prácticas culturales y la apropiación social que están en la base de un modelo de ciudad sostenible y sustentable ambientalmente". Ésta se ha puesto en marcha en relación con conflictos ambientales tales como la mitigación de las emisiones de motos 2 tiempos; contaminación auditiva; recuperación de quebradas y humedales y arbolado urbano.

También y con el liderazgo de Transmilenio y la Policía Metropolitana se lanzó en el mes de junio de 2013 el plan "Transmicultura” que busca transformar aquellos comportamientos que afectan a los usuarios del sistema tales como el vandalismo, transitar por el lado izquierdo, llevar el bolso en la espalda, los hurtos entre otros.

20 SEN, Amartya. La cultura como base del desarrollo contemporáneo. Diálogos. Nueva York: Unesco, 1997.

21 PNUD. Bogotá: una apuesta por Colombia. Informe de Desarrollo Humano 2008. Bogotá: PNUD, 2008, p. 221. 
En correspondencia con todo lo anterior, la Universidad del Rosario, en su Proyecto Educativo Institucional, señala la misión de la Universidad en los siguientes términos: "Impartir una sólida formación ética, humanística y científica que, unida a la investigación y a una idónea y exigente docencia, permita a esta comunidad educativa formar integralmente personas insignes y actuar en beneficio de la sociedad, con un máximo sentido de responsabilidad". ${ }^{22}$

Dentro de sus criterios, el PEI de la Universidad del Rosario establece a Colombia como el horizonte de todos sus esfuerzos, así como la formación integral de sus estudiantes será su propósito fundamental mediante una educación activa y creativa. El PEI afirma que se promoverá la consolidación de su comunidad educativa; que se trabajará en la construcción de una estructura académica enmarcada en el ámbito nacional que permita el enriquecimiento del conocimiento para aportar a la solución de problemas del país y se propiciará el intercambio de experiencias y programas a nivel internacional.

El PEI, en su perspectiva de formación integral, propone a los estudiantes una comprensión de la dimensión intercultural en un mundo globalizado, proporcionando realidades del contexto local y global que permean todos los programas académicos de manera transversal. Igualmente, de acuerdo con lo establecido en el Plan Integral de Desarrollo (PID), la Universidad espera en el 2015 estar en permanente interacción con el medio, tener capacidad para identificar las necesidades y los problemas prioritarios del entorno en sus campos de acción y desarrollar proyectos para aportar a su comprensión y solución. ${ }^{23}$

22 UNIVERSIDAD DEL ROSARIO. Proyecto Educativo Institucional (PEI). Bogotá: Editorial Universidad del Rosario, 2010.

23 PLAN INTEGRAL DE DESARROLLO (PID) 2004-2015. Crecimiento con calidad e identidad. Bogotá: Universidad del Rosario. 
Además, la Universidad tiene como propósito ser reconocida por la pertinencia de su quehacer para avanzar hacia una sociedad justa y en paz e influir en el crecimiento cultural, social y económico del país. Dentro de este enfoque, la Universidad del Rosario hace parte de los eventos en los que se ha venido trabajando la construcción de políticas culturales en la educación superior. Realizó un conversatorio el 29 de octubre del 2008, con la intervención de expertos en gestión y políticas culturales. ${ }^{24}$ Así mismo, participó en la organización del conversatorio con la Universidad Distrital para discutir sobre las políticas culturales desde las universidades ${ }^{25}$ esta intervención trató de poner de presente los distintos factores que han llevado a establecer cambios trascendentales en el escenario cultural de las Universidades. En el 2009 lideró la mesa de políticas culturales del comité Ascun Cultura (nodo Bogotá) y organizó el foro "¿Por qué una política cultural en las universidades?", cuyas memorias se compilaron en el libro Universidad y cultura, reflexiones sobre políticas culturales. ${ }^{26}$

El Plan Integral de Desarrollo de la Universidad 20042019, en el eje de Consolidación de la Identidad Rosarista, implementó la inserción de políticas, estrategias, programas y líneas de acción cuyo foco esté en el concepto de Universi-

24 María del Rosario Bonilla y Raúl Niño quienes presentaron las ponencias: "El papel de la universidad en tanto escenario de la multiculturalidad" y "Políticas culturales en el umbral del conocimiento", respectivamente.

25 Con la ponencia de Johanna Mahuth Tafur, coordinadora académica y administrativa de los posgrados de la Escuela de Ciencias Humanas de la Universidad del Rosario, denominada "Hacia la construcción de una política cultural en la Universidad del Rosario” (2010).

26 DÍAZ TÁMARA, Adriana; GUZMÁN BEJARANO, Margarita. Universidad y cultura: reflexiones sobre políticas culturales. Bogotá: Universidad del Rosario, 2010. 
dad Promotora de Salud, ${ }^{27}$ entendida como "aquella que se compromete con la creación de un entorno y una cultura que fomente la salud y bienestar integral de los miembros que la componen: estudiantes, académicos y funcionarios". ${ }^{28}$ Para la implementación del programa de Universidad Promotora de Salud se diseñaron los ejes de: calidad de vida, permanencia, actividad física, recreación y deporte y cultural.

El eje cultural parte entonces del reconocimiento del papel de la cultura en el fortalecimiento del medio universitario y como el espacio en "donde se escenifican las transformaciones en los hábitos y modos de vida" ${ }^{29}$ de las personas que componen la comunidad universitaria. Este planteamiento define para la cultura un rol estratégico en la apropiación de los pilares sobre los cuales descansa la propuesta de Universidad Promotora de Salud. ${ }^{30}$

Ahora bien, la Universidad del Rosario, gracias a sus 360 años de historia, tiene un patrimonio cultural que es legado de su pasado y que hace parte del ambiente que se vive en la institución. En la última década, la Universidad ha tenido una transformación importante: ha aumentado considerablemente

27 UNIVERSIDAD DEL ROSARIO. Plan Integral de Desarrollo $2004-$ 2019. Bogotá: Editorial de la Universidad del Rosario, 2009, p. 63 y 64.

28 UNIVERSIDAD DEL ROSARIO. Fortalecimiento de la Proyección del Medio Universitario: Universidad Promotora de Salud. Documento final. Bogotá: Universidad del Rosario, 2011.

29 INSTITUTO DISTRITAL DE CULTURA Y TURISMO. Políticas culturales distritales 2004-2016. Bogotá: IDCT, 2004, p. 9; UNIVERSIDAD DEL ROSARIO. Fortalecimiento de la Proyección del Medio Universitario: Universidad Promotora de Salud. Documento final. Bogotá: Universidad del Rosario, 2011.

30 UNIVERSIDAD DEL ROSARIO. Fortalecimiento de la Proyección del Medio Universitario: Universidad Promotora de Salud. Documento final. Bogotá: Universidad del Rosario, 2011. 
el número de estudiantes procedentes de muchos lugares del país y del exterior, y hay una mayor población femenina entre sus estudiantes. En el año 2005 adquirió la sede del norte, se creó la Cancillería, el Centro de Estudios de Turquía y el Centro de Estudios Teológicos y de las Religiones (CETRE) y más recientemente se creó la Unidad de Patrimonio Histórico y Cultural. Dicha Unidad integra, preserva y protege el patrimonio histórico y cultural de la Universidad, mediante la implementación de políticas que permitan la conservación de la memoria institucional y la formulación de estrategias para la divulgación de la cultura e identidad rosaristas. Esta Unidad maneja varias líneas de investigación conformadas por catedráticos de la universidad y por el apoyo de auxiliares del grupo de investigación "Estudios Sociales de las Ciencias, las Tecnologías y las Profesiones".

La Unidad de Patrimonio Histórico y Cultural tiene a su cargo el Archivo Histórico de la Universidad, cuyo espíritu es consolidarse como un Centro de Información que apoya, fomenta y divulga investigación mediante la administración de su acervo documental y bibliográfico con el uso de las nuevas tecnologías, de acuerdo con los estándares internacionales. El archivo histórico fomenta y difunde la investigación, genera espacios de discusión que vinculan las actividades de la Universidad con las colecciones que conserva. Otra de las actividades que realiza este archivo es la conservación y preservación a perpetuidad de las colecciones como parte del patrimonio histórico de la Universidad y del país. Igualmente, realiza alianzas estratégicas que permiten fortalecer las actividades del Archivo mediante el intercambio de experiencias y servicios. Vale decir que también esta unidad dirige la revista Nova et Vetera que, creada en 1905, es una de las siete publicaciones periódicas más antiguas del país y es de gran aceptación por la comunidad 
rosarista. Además, esta Unidad de Patrimonio Cultural e Histórico realiza la curaduría de todo el patrimonio artístico de la Universidad y, para ese efecto, en unión de servicios generales, actualmente desarrolla la restauración del Claustro, patrimonio cultural nacional desde 1975, con el fin de conservar y mantener la edificación.

Todo lo anterior ha implicado una verdadera revolución cultural que, además de crear nuevas dependencias, ha implicado la revisión y actualización de varios conceptos y políticas. Por ejemplo, durante el año 2005 se implementó el núcleo de formación rosarista en todos los programas de pregrado para apoyar la formación integral.

Dando continuidad al proceso de cambio cultural descrito, la Universidad del Rosario pasa entonces de programar la actividad folklórica, a tener dentro de sus asignaturas elementos de formación tales como deportes, bienestar y calidad de vida, problemática social y temas culturales, que hacen parte del propósito de formación integral con el que se encuentra comprometida. Todos estos cambios hacen parte de la dinámica que sirvió de base para iniciar el proceso de construcción de una política cultural en la Universidad.

Para el diseño de la política cultural en la Universidad del Rosario se integró un equipo técnico conformado por funcionarios de la Decanatura del Medio Universitario, la Dirección de Extensión y la Escuela de Ciencias Humanas. Se elaboró un cronograma y se establecieron etapas del proceso que involucraron a directivos, profesores, funcionarios y estudiantes. Dicho proceso se inició en el año 2009 con la ejecución de diferentes acciones: se trabajaron grupos focales con estudiantes, se revisaron indicadores de consumo y prácticas culturales, se llevaron a cabo entrevistas a los directores y funcionarios de la institución 
que han participado en desarrollos culturales y que han tenido la experiencia en la formulación de políticas culturales.

Es el caso de la Dirección de Extensión, ${ }^{31}$ desde donde se reconoce que el tema cultural de la universidad es parte de lo que se presenta en la Red Nacional de Extensión. Desde la Dirección de Extensión se afirma que la gestión cultural es un elemento que está en el entorno, que le da contexto al Rosario, y con esa perspectiva de territorio y cultura la Universidad también construye ciudadanía. La pertinencia de una política cultural en la Universidad del Rosario también se reconoce desde la Escuela de Ciencias Humanas, que tiene una histórica relevancia en el campo de la gestión cultural en Colombia y en la región pues ha desarrollado el primer programa de educación formal de gestión cultural en el país, el segundo en América Latina. La especialización en Gerencia y Gestión Cultural es una de las más importantes y antiguas a nivel nacional en la formación de gestores culturales, ha participado en la construcción de la gestión de lo cultural en el país a través de sus egresados, docentes y acciones de extensión. La construcción de la política se plantea objetivos y dimensiones que tienen que ver con la formación integral y ciudadana, pero también con nuestra propia gestión cultural, con la investigación en cultura, con el fomento del patrimonio material, inmaterial y natural como escenario de construcción de memorias, así como con la importancia de la cultura en el sector productivo.

Por su parte, la Decanatura del Medio Universitario ${ }^{32}$ destaca la importancia de la historia de la institución como

31 Entrevista realizada al director de Extensión, Francisco José Mejía Pardo (agosto de 2012).

32 Entrevista realizada al decano del Medio Universitario, Gabriel Silgado Bernal (marzo de 2012). 
elemento significativo y relevante para la construcción de una política cultural en la Universidad. Igualmente, se reconoce que la riqueza patrimonial representada en el Claustro, la capilla de La Bordadita, el Archivo Histórico y la pinacoteca constituye un valor único que permite desarrollar una formación arraigada en la identidad y la memoria de una institución de 360 años de historia, significativa para el país. Alrededor de todo ese acervo patrimonial, la Decanatura del Medio Universitario viene desarrollando actividades académicas y extracurriculares apoyadas en el núcleo de formación rosarista, que hay que visibilizar y fortalecer a través de una política cultural. Dicha política debe caracterizarse por ser transversal para cumplir con el objetivo de transformar positivamente a aquellas personas que en el futuro serán los líderes de los grandes temas en el desarrollo cultural del país. Así, los egresados de los diferentes programas de la Universidad del Rosario encuentran espacios y oportunidades para contribuir en el diseño e implementación de las políticas culturales a nivel distrital y nacional. 